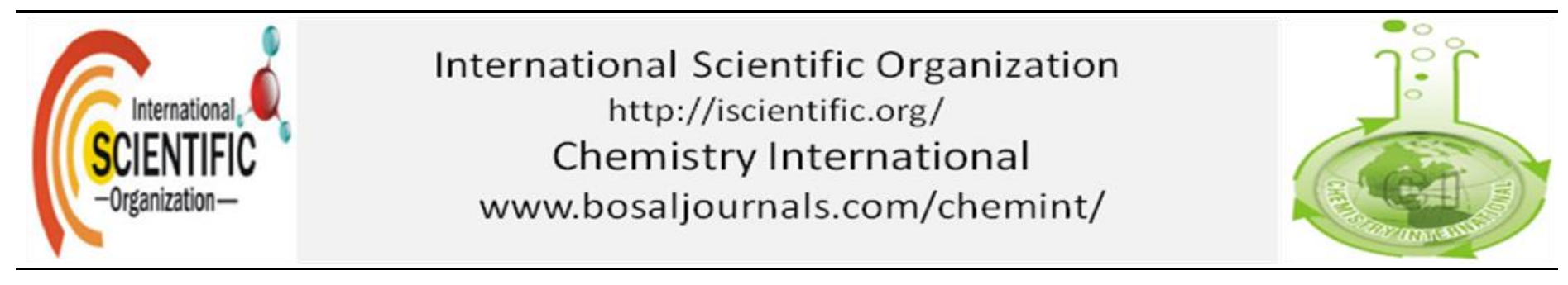

\title{
Basics in colors, dyes and pigments chemistry: A review
}

\author{
Hassan Abazied Shindy \\ Department of chemistry, Faculty of Science, Aswan University, Aswan, Egypt \\ *Corresponding author's E. mail: hashindy2@hotmail.com
}

\section{A R T I C L E I N F O}

\section{Article type:}

Review article

Article history:

Received July 2015

Accepted August 2015

January 2016 Issue

Keywords:

Colors

Dyes

Pigments

Synthesis of dyes

Properties of dyes

Uses of dyes

Classification of dyes

\section{A B S T R A C T}

More than one hundred multi choice problems and their solutions in colors, dyes and pigments chemistry are represented in this paper review. Reviewing the basic, fundamental principles and or the knowledge of colors, dyes and pigments chemistry via multi choice problems and their solutions is an interesting type of reviewing and can be considered as a novel and or patent type of reviewing which is lacking in literature. This paper review is informative and acts as a mordant and or stabilizer for the basic information, fundamental understanding, principles and or knowledge in colors, dyes and pigments chemistry, and it may be very interesting to read by a large community of dyers and colorists, and also by professors and teachers in this field. It is valuable both for getting an overview in the field of colors, dyes and pigments chemistry and as a mine of information for the dyestuff chemists. In addition, this paper review can be published and or printed as a thesis and or note book teaching and lectures for undergraduate and graduate students of chemistry departments and or chemistry institutions at any university. Besides, this paper review can be used and or will be most valuable in domestic and or international scientific and or chemistry competitions, in organic chemistry as general and particularly in the field of colors, dyes and pigments chemistry.

C 2016 International Scientific Organization: All rights reserved.

Capsule Summary: The basics of colors, dyes and pigments chemistry have been reviewed.

Cite This Article As: H. A. Shindy. Basics in Colors, dyes and pigments chemistry: A review. Chemistry International 2(1) (2016) 29-36

\section{INTRODUCTION}

Color has always attracted the attention of the human beings. Defining a color and turning the tint are indeed of paramount importance in many areas such as paintings, printings, clothing, pictures and screens. This task is challenging because human eye is very sensitive to the light frequency. Hundreds of investigations have been carried out over the years in order to elaborate dyes with specific properties for targeted applications (Champagne et al., 2006; Zuppirdi et al., 2001). The color of any colored object comes from the light that it does not absorb. The color of a transparent object is due to the colors of light that can pass through the material.
For example, white light passing through a glass of red wine looks red because the wine has absorbed the other colors, and lets only the red light pass through. Looking through a piece of red cellophane at objects of different colors, all colors but red vanish. This is because the cellophane absorbs light with all other colors except red. The color most strongly absorbed is the complement of the color that passes through the material (Chandrasekaran, 2001).

Dyes are colored ionizing aromatic and/or heterocyclic compounds which show an affinity towards the substrate to which they are being applied. They are generally applied in a solution that is aqueous. Dyes may also require a mordant to improve the fastness of the dye on the material on which it is applied. Basically, dyes are aromatic 
compounds and their structures possess aryl rings that have delocalized electron systems. These structures are said to be responsible for the absorption of electromagnetic radiations that have varying wavelengths, based upon the energy of the electron clouds. It is actually because of this reason that chromophores do not make colored dyes, rather they make the dyes proficient in their ability to absorb radiation. Chromophores act by making energy changes in the delocalized electron cloud of the dye. This alteration invariably results in the compound absorbing radiation within the visible range of colors and not outside it. Human eye detects this absorption and responds to the colors. Another possibility is that if the electrons are removed from the electron cloud, it may result in loss of color. Removing electrons may cause the rest of the electrons to revert to the local orbits. A very good example is the schiffs reagent, as sulphurous acid reacts with pararosaniline a sulphonic group attaches itself to the compounds central carbon atom, this hampers the conjugated double bond system of the quinoid ring and causes the electrons to become localized. As a consequence the ring ceases to be a chromophore, and as a result the dye becomes colorless. To conclude, chromophores are the atomic configurations which have delocalized electrons. Generally, they are represented as carbon, nitrogen, oxygen and sulphur. They can have alternate single and double bonds (Anonymous, 2008). The dyestuff industry was and still remains a vibrant, challenging industry requiring a continuous stream of new products because of the quickly changing world in which we live. The scale and growth of the dye industry have been inextricably linked to that of the textile industry. The rapid growth in the high-tech uses of dyes, particularly in ink-jet printing is beginning to make an impact. Although the volumes of high-tech dyes will remain small in comparison to dyes for traditional applications, the value will be significant because of the higher price of these specialized dyes (Hunger, 2003).

Pigments are colorants that are insoluble in water and most of the solvents and comparatively lesser in number and faster than dyes. Pigment particles are about 1-2 microns in size ( 1 micron $=1 / 1000$ meter $)$, it means that the particles can be seen under a magnifying glass. During application, pigments have the capacity to retain particulate or crystalline structure (Anonymous, 2008b). Pigments usually have special properties that make them ideal for coloring other materials. A pigment must have a high tinting strength relative to the materials it colors. It must be stable in solid form at ambient temperatures. Most pigments used in manufacturing and the visual arts are dry colorants, usually ground into a fine powder. This powder is added to a vehicle (or matrix), a relatively neutral or colorless material that acts as a binder. In some cases, a pigment can be manufactured from a dye by precipitating a soluble dye with a metallic salt. The resulting pigment is called a lake pigment (Anonymous, 2008c).

I recommend this paper review (Bahl and Bahl, 2009; Shindy, 2012) unreservedly to anyone interested in the subject, chemistry libraries, and also in the personal bookshelves of every organic heterocyclic and/or aromatic dye chemists.

\section{Multi-choice problems in colors, dyes and pigment chemistry}

1-The group which leads to intensifying the color degree is called:
a-Auxochromes.
b-Chromophores.
c-Positive groups.

2-Tannic acid, a commercial form of tannin is a weak acid used as a mordant and/or color stabilizer (http://en.wikipedia.org/wiki/Tannic_acid), its structure is:

a-A polyphenol compound.

b-A polyalcohol compound.

c-A polysulphonic compound.

$3-\mathrm{C}=\mathrm{O}, \mathrm{C}=\mathrm{S}, \mathrm{CH}=\mathrm{N}, \mathrm{CH}=\mathrm{CH}$ are:

a-Chromophoric groups.

b-Auxochromic groups.

c-positive groups.

4-p-quinonoid and 0-quinonoid structures are:

a-Auxochromic groups.

b-Chromophoric groups.

c-Negative groups.

5-The weak acidity of tannic acid is due to:

a-Phenolic $\mathrm{OH}$ groups in its structure.

b-Alcoholic $\mathrm{OH}$ groups in its structure.

c-Sulphonic SO3H groups in its structure.

6-Normal daylight or white light is a mixture of all the wavelengths to which we can respond and some to which we cannot, we respond to:

a-Infra-red rays.

b-Ultra-violet rays.

c-Visible light.

7-Normal daylight or white light is a mixture of all the wavelengths to which we can respond and some to which we cannot, human eye responds to the wavelengths between:

a-200nm-300nm.

b-400nm-750nm

c-900nm-1000nm.

8-Lanital fibers belong to:

a-Half synthetic fibers

b-Natural fibers.

c-Synthetic fibers.

9-The dyes are thought to be deeper in color when they absorb light at:

a-Longer wavelength band.

b-Shorter wavelength band.

c-Medium wavelength band.

10-The dyes are thought to be lighter in color when they absorb light at:

a-medium wavelength band.

b-shorter wavelength band.

c-Longer wavelength band. 
11-Prepared by coupling p.nitro benzene diazonium chloride (diazotized p.nitro aniline) with naphthionic acid (1naphthyl amine-4-sulphonic acid):

\section{a-Acid red. \\ b-Chrysoidine. c-Orange II.}

12-Replacing the $2 \mathrm{NH}$ groups in indigotin by sulphur atoms gives:

a-Thioindoxyl.

b-Thioindigotin.

c-Thioindoxylic acid.

13-Replacing the sulphur atoms in thioindigotin by $2 \mathrm{NH}$ groups

gives:

a-Indoxyl.

b-Indoxylic acid.

c-Indigotin.

14-Acid red is prepared by coupling p.nitro benzene diazonium chloride (diazotized p.nitroaniline) with:

a-1-naphthyl amine-4-sulphonic acid.

b-1-naphthyl amine-2-sulphonic acid.

c-1-naphthyl amine-3-sulphonic acid.

15 -We get thioindigotin dye by replacing the $2 \mathrm{NH}$ groups in indigotin dye by:

a-Oxygen atoms.

b-Sulphur atoms.

c-Selenium atoms.

16-We get indigotin dye by replacing the sulphur atoms in thioindigotin dye by:

a-2 $\mathrm{OH}$ groups.

b-2 CO groups.

c-2 NH groups.

17-The color of Congo Red dye in acid/base media is inverse to the color of:

a-Phenolphthaleine dye in acid/base media.

b-Methyl Orange dye in acid/base media.

c-Litmus paper in acid base media.

18-The silver bromide emulsion in photographic films or plates is sensitive and/or affected only by light of wavelengths in the range of:

a-350 to 530 nanometres.

b-550 to 650 nanometers.

c-750 to 850 nanometers,

19-Because its color remains even after extensive dilution, it

is used to trace pollution of water supplies by sewrage:

a-Fluorescein.

b-Eosin.

c-Erythrosine.

20-The trade name of 2,4-di nitroso resorcinol dye is:

a-Green resorcin.

b-Green naphthol.

c-Yellow naphthol.

21-Used as a mild purgative:

a-Rhodamine $\mathrm{B}$.

b-Fluorescein.

c-Eosin.

22-The trade name of 1-nitroso-2-naphthol dye is:
a-Yellow naphthol.

b-Green naphthol.

c-Picric acid.

23-In the synthesis of triarylmethane dyes, the leuco base compound converted to the color base compound by:

a-Sulphonation.

b-Oxidation.

c-Reduction.

24-The trade name of 2,4,6-tri nitro phenol dye is:

a-Green naphthol.

b-Yellow naphthol.

c-Picric acid.

25-In the preparation of triarylmethane dyes, the color base compound converted to the dye by:

a-Acidification.

b-Alkalization.

c-Nitration.

26-Green resorcin dye is prepared by entering:

a-2 Nitroso groups to resorcinol.

b-2 Nitro groups to resorcinol.

c-2 Amino groups to resorcinol.

27-In the synthesis of triarylmethane dyes, the dye can be reconverted to the color base compound by:

a-Chlorination.

b-Alkalization.

c-Acidification.

28-Green naphthol dye is synthesized by entering:

a-One nitro group to B-naphthol.

b-One amino group to B-naphthol.

c-One nitroso group to B-naphthol.

29-The trade name of 2,4-diamino-azo benzene dye is:

a-Chrysoidine.

b-Acid red.

c-Orange II.

30-Picric acid can be prepared by entering:

a-3 Nitroso groups to phenol.

b-3 Nitro groups to phenol.

c-3 Amino groups to phenol.

31-Green resorcin dye is prepared by entering 2 nitroso groups to:

a-Catechol.

b-Quinol.

c-Resorcinol.

32-Rhodamine dyes are:

a-Red cationic dyes

b-Red anionic dyes.

c-Red zwitter ion dyes.

33-Green naphthol dye is synthesized by entering one nitroso group to:

a-1-naphthol.

b-2-naphthol.

c-Phenol.

34-Phthaleine dyes are:

a-Cationic dyes.

b-Anionic dyes.

c-Zwitter ion dyes.

35-Picric acid is synthesized by entering 3 nitro groups to: 

a-Phenol ring.
b-Naphthol ring.
c-Aniline ring.

36-Yellow naphthol dye (naphthol Yellow S) is prepared by: a-Sulpohnation of 1-naphthol followed by nitration. b-Nitration of 1-naphthol followed by sulphonation. c-Iodination of 1-naphthol.

37-Used as extremely powerful laxative:

a-Mercurochrome.

b-Phenolphthaleine.

c-Phenolphthalene sulphone.

38-In the preparation of triarylmethane dyes, the color base compound can be reconverted to the leuco base compound by:

a-Oxidation.

b-Reduction.

c-Brominatin.

39-Flouresceine used as:

a-A mild purgative.

b-Antiseptic agents.

c-Antitumor agents.

40 -Dobener violet dye can be synthesized by reaction of benzaldehyde ( 1 molecule) with 2 molecules of:

a-Aniline in the presence of dehydrating agents.

$\mathrm{b}-\mathrm{N}$-methyl aniline in the presence of dehydrating agents.

$\mathrm{c}-\mathrm{N}, \mathrm{N}$-dimethyl aniline in the presence of dehydrating agents.

41-Yellow naphthol dye (naphthol Yellow S) is prepared by: a-Sulpohnation of 1-naphthol followed by nitration. b-Sulpohnation of 2-naphthol followed by nitration. c- Sulpohnation of phenol followed by nitration.

42-The di sodium salt of phenolphthaleine has:

a-Two resonance forms.

b-Three resonance forms.

c-Four resonance forms.

43-The di sodium salts of flouresceine have:

a-Two resonance forms.

b-Three resonance forms.

c-Four resonance forms.

44-The chromophore in dobener violet dye is:

a-o-Quinonoid structure.

b-p-Quinonoid structure.

c-Azo group.

45-The auxochrome in dobener violet dye is:

a-NH2 group.

$\mathrm{b}-\mathrm{OH}$ group.

c-NHCH3 group.

46-Rhodamine B. has:

a-Two resonance forms.

b-Three resonance forms.

c-Four resonance form.s

47-Alizarin dye is:

a-Cationic dye.

b-Anionic dye.

c-Zwitter ion dye.

48-Used in their reduced state:
a-Vat dyes.

b-Azo dyes.

c-Azine dyes.

49-Transfer of dye from dye bath to the fiber named:

a-Levelness.

b-Exhaustion.

c-Shading.

50-The sodium salts of sulphonic and carboxylic acids are: a-Basic dyes.

b-Vat dyes.

c-Acid dyes.

51-Insoluble azo dyes are:

a-Azoic dyes.

b-Water insoluble cationic dyes.

c-Sodium salts of sulphonic and carboxylic acids.

52-Anionic dyes are:

a-Cationic dyes.

b-Acidic dyes.

c-Zwitter ion dyes.

53-Cationic dyes are:

a-Anionic dyes.

b-Basic dyes.

c-Zwitter ion dyes.

54-Yellow naphthol dye ( Naphthol yellow S) is used in: a-Pharmaceutical industry.

b-Coloring foods.

c-Coloring woods.

55-Reaction of 1-ethyl quinolinium iodide salt with 1-ethyl

quinaldinium iodide salt in alcohol/base gives:

a-Pinacyanol dye,

b-Kryptocyanine dye

c-Ethyl red dye.

56-Used essentially as photographic sensitizers:

a-Cyanine dyes.

b-Azine dyes.

c-Phthaleine dyes.

57-Reaction of 2 mole of 1-ethyl quinaldinium iodide salt with triethylorthoformate in alcohol/base gives:

a-Dimethine cyanine dye.

b-Trimethine cyanine dye.

c-Monomethine cyanine dye.

58-Reaction of 2 mole of 1-ethyl lepidinium iodide salt with triethylorthoformate in alcohol/base gives:

a-Apocyanine dye.

b-Styryl cyanine dye.

c-Trimethine cyanine dye.

69-The trade and/or famous name of 2,2-trimethine cyanine dye is:

a-Ethyl red.

b-Pinacyanol.

c-Kryptocyanine.

60-The trade and/or famous name of 1,2-dihydroxy anthraquinone dye is:

a-Green resorscen.

b-Green nahthol.

c-Alizarin.

61-Methyl orange is used as: 
a-Antitumor agent.

b-Indicator in analytical chemistry.

c-Coloring woods.

62-Mercurochrome is used as:

a-Anticancer agent.

b-Antiseptic agent.

c-Coloring food.

63-Chrysoidine dye is:

a-2,4-dihydroxy azo benzene.

b-2,4-dimethoxy azo benzene.

c-2,4-diamino azo benzene.

64-Alizarine dye is:

a-1,2-dimethyl anthraquinone.

b-1,2-dihydroxy anthraquinone.

c-1,2-diethoxy anthraquinone.

65-Prepared by coupling of diazonium salt of sulphanilic acid with B-naphthol:

a-Orange II.

b-Acid red.

c-Methyl orange.

66-Used for coloring woods:

a-Congo red dye.

b-Besmark brown dye.

c-Crystal violet dye

67-The scientific name of green resorcin dye is:

a-2,4-Di nitro resorcinol.

b-2,4-Di nitroso resorcinol.

c-2,4-2,4-Di azo resorcinol.

68-Prepared by coupling benzene diazonium chloride with m.phenylenediamine:

a-Chrysoidine dye.

b-Congo red dye.

c-Green naphthol dye.

69-Prepared by condensing benzaldehyde with 2 molecules

of N,N-dimethyl aniline:

a-Pararosaniline dye.

b-Malachite green dye.

c-Gentian violet dye.

70-Synthesized by condensing p.amino benzaldehyde with 2 molecules of aniline:

a-Crystal violet dye.

b-Dobner violet dye.

c-Pararosaniline dye.

71-Prepared by condensing of p.amino benzaldehyde with one molecule of o.toluidine and one molecule of aniline:

a-Roseaniline dye.

b-Phenolphthaleine dye.

c-Fluorescein dye.

72-Synthesized by reaction of dimethylaniline ketone

(Mischlers ketone) with N,N-dimethyl aniline:

a-Eosin dye.

b-Crystal violet dye.

c-Erythrosine dye.

73-Prepared by reaction of aniline ketone (p,pdiaminodiphenyl ketone) with $\mathrm{N}, \mathrm{N}$-dimethyl amino benzene: a-Rhodamine B dye.

b-Gentian violet dye.
c-Mercurochrome dye.

74-NH2 and N(CH3)2 groups are the auxochromes in:

a-Gentian violet dye.

b-Phenolphthaleine sulphone dye.

c-Pinacyanol dye.

75-N(CH3)2 groups are the auxochromes in:

a-Ethyl red dye.

b-Crystal violet dye.

c-Kryptocyanine dye.

76-Synthesized by coupling tetrazotized benzidine with 2

molecules of 1-naphthylamine-4-sulphonic acid:

a-Congo red dye.

b-Besmark brown dye,

c-Methyl orange dye.

77-Congo red dye is prepared by coupling tetrazitized

benzidine with 2 molecules of:

a-1-naphthylamine-2-sulphonic acid.

b-1-naphthylamine-3-sulphonic acid.

c-1-naphthylamine-4-sulphonic acid.

78-Prepared by coupling tetrazotized m.phenylenediamine with 2 molecules of m.phenylenediamine:

a-Acid red dye.

b-Besmark brown dye.

c-Chrysoidine dye.

79-Synthesized by condensing phthalic anhydride with phenols in the presence of dehydrating agents:

a-Azo dyes.

b-Azine dyes.

c-Phthaleine dyes.

80-Prepared by mercuration of dibromo fluorescein followed by action of $\mathrm{NaOH}$ :

a-Fluorescein dye.

b-Mercurochrome dye.

c-Phenolphthaleine dye.

81-Synthesized by bromination of dibromo fluorescein

followed by action of $\mathrm{NaOH}$ :

a-Eosin dye.

b-Erythrosine dye.

c-Fluorescein dye.

82-Used as extremely powerful laxative:

a-Mercurochrome.

b-Indigotin.

c-Phenolphthaleine.

83-Used as antiseptic agents:

a-Mercurochrome.

b-Malachite green.

c-Green resorcine.

84-Tetra iodo fluorescein is:

a-Erythrosine .

b-Eosin.

c-Rhodamine B.

85-Used in pharmaceutical industry:

aFluorescein.

b-Eosin.

c-Erythrosine. 
86-Condensing phthalic anhydride (1 molecule) with 2 molecules of m.aminophenol or its derivatives in the presence of dehydrating agents gives:

a-Rhodamines.

b-Phthaleines.

c-Fluoresceins.

87-Condensing phthalic anhydride (1 molecule) with 2 molecules of $\mathrm{N}, \mathrm{N}$-diethylm.aminophenol in the presence of $\mathrm{ZnCl} 2$ gives:

a-Eosin.

b-Chrysoidine.

c-Rhodamine B.

88-Condensing phthalic anhydride (1 molecule) with 2 molecules of resorcinol in the presence $\mathrm{H} 2 \mathrm{SO} 4$ gives:

a-Phenolphthaleine.

b-Fluorescein.

c-Erythrosine .

89-Used in preparing Schiff reagents:

a-Roseaniline.

b-Malachite green.

c-Gentian violet.

90-Indigotin dye is used in:

a-Plastic industry.

b-Photographic industry.

c-Manufacturing of printing inks.

91-Crystal violet dye is used in:

a-Pharmaceutical industry.

b-Manufacturing of printing inks.

c-polymer industry.

92-Erythrosine is used in:

a-Coloring foods.

b-Coloring woods.

c-Detergents industry.

93-Used in explosive industry:

a-Yellow naphthol.

b-Picric acid.

c-Orange II.

94-Congo red is used in:

a-Fiber industry.

b-Analytical chemistry as indicator.

c-Medicine as antitumor agents.

95-Condensing phthalic anhydride with 2 molecules of phenol in the presence of dehydrating agents gives:

a-Phenolphthaleine.

b-Phenolphthaleine sulphone.

c-Eosin.

96-The scientific name of yellow naphthol dye (Naphthol yellow $S$ dye) is:

a-2,4-dinitro-1-naphthol-7-sulphonic acid.

b-2,4-dinitroso-1-naphthol-7-sulphonic acid.

c-2,4-diazoo-1-naphthol-7-sulphonic acid.

97-Reaction of benzaldehyde ( 1 molecule) with 2 molecules

of aniline in the presence of dehydrating agents gives:

a-Gentian violet dye.

b-Dobener violet dye.

c-Crystal violet dye.
98-Sulphonation of 1-naphthol followed by controlled nitration gives:

a-Green naphthol dye.

b-Yellow naphthol dye (Nphthol yellow S dye). c-Acid red dye.

99-Yellow naphthol dye (Naphthol yellow S dye) prepared by:

a-Sulphonation of 1-naphthol followed by acetylation.

b-Sulphonation of 1-naphthol followed by alkylation.

c-Sulphonation of 1-naphthol followed by nitration.

100-The trade and/or famous name of 4,4-trimethine cyanine dye is:

a-Pinacyanol.

b-Kryptocyanine dye.

c-Ethyl red dye.

101-Reaction of 1-ethyl quinolinium iodide salt with 1-ethyl lepidinium iodide salt in alcohol/base gives:

a-2,2-monomethine cyanine dye.

b-2,4-monomethine cyanine dye.

c-4,4-monomethine cyanine dye.

102-Reaction of 1-ethyl quinolinium iodide salt with 1-ethyl quinaldinium iodide salt in alcohol/base gives:

a-2,2-monomethine cyanine dye.

b-2,4-monomethine cyanine dye.

c-4,4-monomethine cyanine dye.

103-The silver chloride emulsion in photographic films or plates is sensitive and/or affected only by light of wavelengths in the range of:

a-350 to 450 nanometres.

b-550 to 650 nanometers.

c-750 to 850 nanometers,

104-The property of substance to change color due to a change in temperature called:

a-Halochromism.

b-Solvatochromism.

c-Thermochromism.

105-Occurrence of a change in the color of a chemical substance due to a change in solvent polarity called:

a-Solvatochromism.

b-Thermochromism.

c-Halochromism.

106-The property of substance to change color due to a change in the $\mathrm{pH}$ media (hydrogen ion concentration) called: a-Thermochromism.

b-Halochromism.

c-Solvatochromism.

\section{The solutions}

1-a-Auxochromes.

2- a-A polyphenol compound.

3-a-Chromophoric groups.

4-b-Chromophoric groups.

5-a-Phenolic $\mathrm{OH}$ groups in its structure.

6-c-Visible light.

7-b-400nm-750nm. 
8-a-Half synthetic fibers.

9-a-Longer wavelength band.

10-b-Shorter wavelength band.

11-a-Acid red.

12-b-Thioindigotin.

13-c-Indigotin.

14-a-1-naphthyl amine-4-sulphonic acid.

15-b-Sulphur atoms.

16-c-2NH groups.

17-c-Litmus paper in acid/base media.

18-a-350 to 530 nanometers.

19- a-Fluorescein.

20-a-Green resorcin.

21-b-Fluorescein.

22-a-Green naphthol.

23-b-Oxidation.

24-c-Picric acid.

25-a-Acidification.

26-a-2 Nitrozo groups to resorcinol.

27-b-Alkalization.

28-c-One nitrozo group to B-naphthol.

29-a-Chrysoidine.

30-b-3 nitro groups to phenol.

31-c-Resorcinol.

32-a-Red cationic dyes.

33-b-2-naphthol.

34-b-Anionic dyes.

35-a-Phenol ring.

36-a-Sulphonation of 1-naphthol followed by nitration.

37-b-Phenolphthaleine.

38-b-Reduction.

39-a-Amild purgative.

40-a-Aniline in presence of dehydrating agent.

41-a-Sulphonation of 1-naphthol followed by nitration.

42-a-Two resonance forms.

43-c-Four resonance forms.

44-b-p-Quinonoid structure.

45-a-NH2 group.

46-c-Four resonance form.

47-b-Anionic dye.

48-a-Vat dyes.

49-b-Exhaustion.

50-c-Acid dyes.

51-a-Azoic dyes.

52-b-Acidic dyes.

53-b-Basic dyes.

54-b-Coloring foods.

55-c-Ethyl red dye.

56-a-Cyanine dyes.

57-b-Trimethine cyanine dye.

58-c-Trimethine cyanine dye.

59-b-Pinacyanol.

60-c-Alizarin.

61-b-Indicator in analytical chemistry.

62-b-Antiseptic agents.

63-c-2,4-diamino azo benzene.

64-b-1,2-dihydroxy anthraquinone.
65-a-Orange II.

66-b-Besmark brown dye.

67-b-2,4-Di nitroso resorcinol.

68-a-Chrysoidine dye.

69-b-Malachite green dye.

70-c-Pararosaniline dye.

71-a-Roseaniline dye.

72-b-Crystal violet dye.

73-b-Gentian violet dye.

74-a-Gentian violet dye.

75-b-Crystal violet dye.

76-a-Congo red dye.

77-c-1-naphthylamine-4-sulphonic acid.

78-b-Besmark brown dye.

79-c-Phthaleine dyes.

80-b-Mercurochrome dye.

81-a-Eosin dye .

82-c-Phenolphthaleine.

83-a-Mercurochrome.

84-a-Erythrosine.

85-c-Erythrosine.

86-a-Rhodamines.

87-c-Rhodamine B.

88-b-Fluorescein.

89-a-Roseaniline.

90-c-Manufacturing of printing inks.

91-b-Manufacturing of printing inks.

92-a-Coloring foods.

93-b-Picric acid.

94-b-Analytical chemistry as indicator.

95-a-Phenolphthaleine.

96-a-2,4-dinitro-1-naphthol-7-sulphonic acid.

97-b-Dobener violet dye.

98-b-Yellow naphthol dye (Nphthol yellow S dye).

99-c-Sulphonation of 1-naphthol followed by nitration.

100-b-Kryptocyanine dye.

101-c-4,4-monomethine cyanine dye.

102-b-2,4-monomethine cyanine dye.

103- a-350 to 450 nanometres.

104-c-Thermochromism.

105-a-Solvatochromism.

106-b-Halochromism

\section{CONCLUSIONS}

The developments of color theories in addition to dyes and pigments synthesis and their applications in textile and non textile multidisciplinary area are growing continuously and rabidly. Certainly, this will makes the present and/or the future of this branch of organic chemistry (colors, dyes and pigments chemistry) is effective, fruitful and very bright.

\section{ACKNOWLEDGEMENTS}

I am thankful to the Chemistry department, Faculty of Science, Aswan University, Aswan, Egypt for supporting this work. 


\section{REFERENCES}

Anonymous, 2008a. Dyes and pigments. http://www.dyespigments.com/what-is-dye.html (accessed July, 27, 2008).

Anonymous, 2008b. Dyes and pigments. http://www.dyespigments.com/difference-niomentsdyes.html (accessed July, 27, 2008).

Anonymous, 2008c. Pigment-Wikipedia, the free encyclopedia: http://en.wikipedia.org/wiki/pigment (Accessed, October, 7, 2008)

Bahl, A., Bahl, B.S., 2009. Advanced organic chemistry, 11391161, (S. Chand, Company LTD, Ram Nagar, New Delhi110 055).

Champagne, B., Guillaume, M., Zutterman, F., 2006. TDDFT investigations of the optical properties of cyanine dyes. Chemical Physics Letters 425(1-3), 105-109.

Chandrasekaran, J., 2001. General article, chemistry of Colors, Resonance, 66-75.

Finar I.L., 1990. Organic Chemistry, The fundamental principles, Volume 1, sixth edition, Pp 868-899.

Hunger, K., 2003. Industrial dyes chemistry, properties, applications, Wiley-VCH.

Shindy, H.A., 2012. Basics, mechanisms and properties in the chemistry of cyanine dyes: A review paper. Mini Reviews in Organic Chemistry 9(4), 352-360.

Shindy, H.A., 2012. Multi Choice Questions and their Answers in Color, Dyes and Pigments Chemistry: A Review Paper, Mini-Reviews in Organic Chemistry 9 (4), 361-373.

Tanic acid: http://en.wikipedia.org/wiki/Tannic_acid (accessed November, 25, 2009).

Zuppirdi, L., Bussac, M.N., 2001. Traite des couleurs, Presses Polytechniques et Universitaires Romandes, Lausanne.

Visit us at: http://bosaljournals.com/chemint/

Submissions are accepted at: editorci@bosaljournals.com 